\title{
A Modified Structural Model for Credit Risk - Utility Indifference Valuation
}

\author{
Gechun Liang * \\ Oxford-Man Institute of Quantitative Finance, \\ University of Oxford, \\ Oxford, OX1 4EH, U.K. \\ and \\ Mathematical Institute, \\ University of Oxford, \\ Oxford, OX1 3LB, U.K. \\ Lishang Jiang \\ Risk Management Institute, \\ Mathematical Department, \\ Tongji University, \\ Shanghai, 200092, China
}

First version: December 10th 2008

Second version: April 10th 2009

${ }^{*}$ The corresponding author, Tel: +4401865 616636 ,

E-mail: liangg@maths.ox.ac.uk 


\begin{abstract}
This paper modifies the classical structural models for credit risk by embedding them into the framework of optimal portfolio problems in an incomplete market. The price of corporate bonds is derived based on the indifference between the investor's two utility maximization problems. Besides the uncertainty of the firm's asset value, we introduce another diffusion process, which comes from the firm's stock value, to drive credit risk. This results in the different behaviors of the credit spread from classical structural models. Two new parameters are introduced into the model, namely the investor's risk aversion parameter and the correlation coefficient between the firm's assets and the stocks it issues, which results in the nonlinearity of the pricing rule. Under the Markovian framework, default occurring at the maturity and default occurring at the first-passage-time are considered, and the corresponding closed formulae are derived by solving the HJB equation Cauchy problem and the HJB equation Dirichlet problem respectively.
\end{abstract}

Keyword: Credit risk, Structural models, Credit spread, Utility indifference valuation, HJB equations. 


\section{Introduction}

In this paper we consider the credit risk of corporate bonds issued by a firm. Traditionally, there are mainly two types of models widely used: structural (or firm value) models and reduced-form (or intensity) models. In structural models, default is determined from the evolution of the firm's structural variables such as assets and liabilities, and occurs if the firm's assets are insufficient according to some measure. See Merton (1974), Black and Cox (1976), Longstaff and Schwartz (1995), Briys and de Varenne (1997), et cetera. Reduced-form models are silent about why a firm defaults, and instead the dynamics of default are exogenously given through a default rate, i.e. the default intensity. See Jarrow and Turnbull (1995), Lando (1998), Duffie and Singleton (1999) and so forth. Our work is based on structural models in this paper.

The basis of structural models, which goes back to Merton (1974), is built on the premise that there is a fundamental process interpreted as the value of the firm's assets. The corporate bonds are regarded as contingent claims on the firm's assets, and default is triggered if the firm is under financial distress, i.e. the firm's asset value falls below its liability value. Black and Cox (1976) introduced safety covenants into bond indenture provisions that give bondholders the right to reorganize a firm if its asset value falls below some nominal barrier, i.e. the first-passage-time model. A lot of research focuses on the extension of first-passage-time models. For example, Longstaff and Schwartz (1995) considered stochastic interest rates, while Briys and de Varenne (1997) introduced stochastic default barriers into first-passage-time models. However, there is a common problem in structural models: the short term credit spread is close to zero if the firm is not in financial distress near the maturity, which contradicts the empirical evidence (See Bielecki and Rutkowski (2002)). The reason is that default can be predicated in structural models if the firm's asset value follows a diffusion process. In fact, this is one of the reasons people introduce jump-diffusion processes into structural models, for example Zhou (1997) and Hilberink and Rogers (2002), or reduce to intensity models by modeling the dynamics of default as jump processes directly.

There is an implicit, but critical, assumption in structural models, namely the uniqueness of equivalent martingale measures. Most of the literature on credit risk focuses on the pricing issues and postulates this assumption without questioning it. Notwithstanding, since it is hard to define a meaningful process for the firm's asset value, let alone observe and trade it (See Schönbucher (2003)), the no-arbitrage argument is not sufficient to deter- 
mine a unique price ${ }^{1}$. In other words, we cannot get the corporate bond price uniquely, because the credit risk, which arises from the collapse of the firm's asset value, cannot be hedged by the underlying. However, the introduction of incompleteness will avoid this problem. Based on this argument, we relax the assumption of the uniqueness of equivalent martingale measures, and modify the classical structural models for credit risk by embedding them into the framework of optimal portfolio problems in an incomplete market.

In incomplete markets, one of the most important issues is how to choose an optimal one among the equivalent martingale measures, and there are mainly two distinct, but intimately related, methods. One is derived from the idea of minimizing the distance between measures according to some criterion. Föllmer and Sondermann (1986) considered the variance optimal measure, while Avellaneda (1998) used the minimal relative entropy measure. Recently, Hobson (2004) generalized these concepts to the $q$-optimal measure, which minimizes the $q$ th moment of the Radon-Nikodym density of the pricing measure with respect to the original real-world measure. The other is called utility indifference valuation, and is more economically motivated. In fact it originates from optimal portfolio problems in incomplete markets. Instead of choosing an equivalent martingale measure for valuation, the mechanism is to find a price at which the buyer (or writer) of a derivative is indifferent in terms of the maximum utility of holding and of not holding the derivative. The approach was initiated by Hodges and Neuberger (1989) and further extended by Davis (1997). Under the Markovian framework, Henderson (2002) provided the solutions for power utilities and exponential utilities respectively, in which the pricing formula for exponential utility is derived and also expansions for the price under power utility. Musiela and Zariphopoulou (2004) solved also for the exponential utility price, while Zariphopoulou (2001) solved a slightly different problem under power utility. For a general overview, we refer to Henderson and Hobson (2004).

Our aim is to adopt the second approach, utility indifference valuation, to model the credit risk of corporate bonds in an incomplete market framework. Bielecki et al (2004) considered the corporate bond pricing in meanvariance portfolio problems, whereas Sircar and Zariphopoulou (2006) and (2007) studied the credit derivative pricing issues by utility indifference valuation. All their works are based on reduced-form models, so the default mechanism is ignored, and the pros of the structural model's economic meanings are sacrificed. Leung et al (2007) tried to apply utility indifference pricing to structural models. However the recovery rate was not considered in their

\footnotetext{
${ }^{1}$ In structural model literature, people often assume there exists such a tradeable asset in markets corresponding to the firm's asset.
} 
model, i.e. the investor will get nothing if default happens. In this paper, we make use of structural models, which are modified by utility indifference valuation, and consider the contracts with recovery rate. Our work can be regarded as a generalization of the classical structural models, as our model can recover the classical structural models. Compared with Leung et al (2007), we consider both default happening at the maturity and default happening at the first-passage-time, and we also consider the recovery rate in the contracts. Even though the underlying processes are diffusion processes rather than jump processes, the credit spread in our model can behave very realistic especially near the maturity, i.e. it is not necessarily nought near the maturity. In fact, the flexibility of the credit spread in our model is due to the introduction of the incompleteness and more realistic contract assumptions.

As the classical structural models, we regard corporate bonds as contingent claims on the firm's assets, which are not tradeable. Nevertheless, the stocks issued by the firm, which are inextricably linked with the firm's assets, are traded by an investor, and the investor will choose an optimal trading strategy so as to maximize his expected utility of terminal wealth. Then the price of corporate bonds is such that the bonds are indifferent to the investor whether he holds them or not. The investor's attitude towards risk is modeled by CARA utility functions as well as variable-separable utility functions. We consider two classical problems: default occurring at the maturity and default occurring at the first -passage-time. Employing the dynamic programming principle (or Bellman principle) and the viscosity solution argument, we derive the Hamilton-Jacobi-Bellman (HJB) equation Cauchy problem and the HJB equation Dirichlet problem respectively. We further obtain the closed formulae by deriving the Monge-Ampère equations and solving them by the Cole-Hopf transformation and the Green's function. The default probability of corporate bonds as well as the hedging strategy of them are also derived.

In our model, the value of corporate bonds will be influenced by the stock price, while the latter is much easier to observe in markets than the firm's asset value. The investor's attitude towards risk will also influence the price, and because of the risk aversion of the investor, the pricing rule is nonlinear, i.e. the value of a portfolio contract is not the same as the sum of the values of its components. Furthermore, under the CARA utility function, we specify this nonlinear relation and derive the corresponding pricing formula in the martingale form. Based on this martingale form, some new features of our model distinctive from the classical structural models are obtained. For example, the credit spread of corporate bonds is not necessarily nought even though the firm is not in financial distress near the maturity. As explained above, default is unlikely to happen in this situation in classical structural models. Finally we recover the classical structural models by proving that 
they are the limiting cases of our model.

The paper is organized as follows: we present the structural model under the utility indifference valuation framework and derive the closed formulae under CARA utility functions and variable-separable utility functions in Section 2. Based on the closed formulae, we further obtain the default probability and the hedging strategy of corporate bonds in Section 3. In Section 4 , we consider the cases of default occurring at the first-passage-time. The comparison with the classical structural models are studied in Section 5, and numerical results are presented in Section 6. We conclude our paper in Section 7 .

\section{$2 \quad$ Utility indifference valuation framework}

In this section we will modify the classical structural models by embedding them into the framework of optimal portfolio problems in incomplete markets, and derive the price of corporate bonds based on utility indifference valuation.

\section{$2.1 \quad$ The model}

To start with, we present several basic assumptions for this paper.

\section{Assumption 2.1 (the market)}

Let $(\Omega, \mathcal{F}, \mathbb{P})$ be a complete probability space. The market is built with three assets: risk-free bonds with interest rate $r$, stocks and corporate bonds, where the latter two assets are issued by a firm. Let $S_{t}$ be the value of the stocks at time $t$ which satisfies

$$
\left\{\begin{array}{l}
d S_{t}=\mu S_{t} d t+\sigma_{S} S_{t} d W_{t}^{S}, \quad t \geq 0 \\
S_{0}=s
\end{array}\right.
$$

where $\mu$ and $\sigma_{S}$ represent the appreciation rate and the volatility rate of the stocks respectively, and $\left(W_{t}^{S}\right)_{t \geq 0}$ is the Brownian motion with its natural filtration $\left\{\mathcal{F}_{t}^{S}\right\}_{t \geq 0}$.

\section{Assumption 2.2 (the firm)}

The firm's asset value, denoted by $\left(V_{t}\right)_{t \geq 0}$, can be observed from the firm's balance sheet, but cannot be traded in markets. It satisfies

$$
\left\{\begin{array}{l}
d V_{t}=\nu V_{t} d t+\sigma_{V} V_{t} d W_{t}^{V}, \quad t \geq 0, \\
V_{0}=v
\end{array}\right.
$$


where $\nu$ is the surplus of the expected return of the firm, i.e. the expected return subtracted by dividends, and $\sigma_{V}$ is the volatility rate of the firm's asset value. $\left(W_{t}^{V}\right)_{t \geq 0}$ is the Brownian motion which generates the filtration $\left\{\mathcal{F}_{t}^{V}\right\}_{t \geq 0}$. The value of the firm's assets and the firm's stocks are correlated, and their correlation coefficient is given by

$$
\operatorname{Cov}\left(d W_{t}^{S}, d W_{t}^{V}\right)=\rho d t \text { with } 0 \leq \rho \leq 1 .
$$

Let $\mathcal{F}_{t}=\mathcal{F}_{t}^{S} \vee \mathcal{F}_{t}^{V}$, and consider the problem on the filtered probability space $\left(\Omega, \mathcal{F},\left\{\mathcal{F}_{t}\right\}_{t \geq 0}, \mathbb{P}\right)$ from now on.

Assumption 2.3 (the contract of corporate bonds)

As the first case, consider the corporate bond, whose face value is $F$, can only be settled at the maturity $T$, no matter when default occurs. If denote by $P_{t}$ the value of the corporate bond, then the investor will get $P_{T}=\min \left(V_{T}, F\right)$ from the corporate bond at the maturity $T$.

Assumption 2.4 (the investor)

The investor has a utility function $U:(a, \infty) \rightarrow \mathbb{R}$, which is increasing, strictly concave, continuously differentiable, and satisfying Inada's conditions:

$$
U^{\prime}(a)=\lim _{x \rightarrow a} U^{\prime}(x)=\infty, \quad U^{\prime}(\infty)=\lim _{x \rightarrow \infty} U^{\prime}(x)=0,
$$

where $a=0$ or $a=-\infty$.

\section{Assumption 2.5 (the trading strategies)}

The investor with initial wealth $x$ will choose a self-financing trading strategy in order to maximize his utility with respect to terminal wealth. Besides trading the stocks, he has two choices, either holding corporate bonds until maturity $T$ or not holding them. Let $\left(\pi_{t}\right)_{0<t<T}$ be the amount invested in stocks. Under the assumption that the trading strategy is self-financing, the wealth equation of the investor is

$$
\left\{\begin{array}{l}
d X_{t}^{\pi}=\left(r X_{t}^{\pi}+\theta_{S} \pi_{t} \sigma_{S}\right) d t+\pi_{t} \sigma_{S} d W_{t}^{S}, \quad t \in(0, T] \\
X_{0}=x
\end{array}\right.
$$

where $\theta_{S}=(\mu-r) / \sigma_{S}$ is the Sharpe ratio of the stocks.

Define the space for the trading strategy $\pi$ as

$$
\begin{aligned}
\Pi[0, T]= & \left\{\pi \mid \pi \in \mathcal{H}^{2} \text { i.e. } \pi_{t} \text { is } \mathcal{F}_{t^{-}} \text {adapted such that } E\left[\int_{0}^{T} \pi_{t}^{2} d t\right]<\infty,\right. \\
& \text { and } \pi \text { is valued on a convex and compact space } \Pi \subset \mathbb{R} .\}
\end{aligned}
$$


Next, we introduce two optimal portfolio problems via which the utility indifference price of the corporate bonds will be derived. The first problem arises from the classical Merton's problem, where the investor doesn't take corporate bonds into account, i.e.

$$
\tilde{V}(x, t)=\sup _{\left(\pi_{s}\right)_{t \leq s \leq T}} E\left[U\left(X_{T}^{\pi}\right) \mid X_{t}=x\right] .
$$

In the second situation, besides trading stocks and risk-free bonds, the investor purchases corporate bonds as well, and he will hold them until the maturity to get $P_{T}=\min \left(V_{T}, F\right)$. Therefore, the investor's value function is

$$
\bar{V}(x, v, t)=\sup _{\left(\pi_{s}\right)_{t \leq s \leq T}} E\left[U\left(X_{T}^{\pi}+P_{T}\right) \mid X_{t}=x, V_{t}=v\right] .
$$

Under the choice of the space for the trading strategy $\Pi[0, T]$, both $(5)$ and (6) are well-posed. Then the utility indifference price of corporate bonds will be given through the two value functions as follows:

Definition 2.1 (Utility Indifference Price)

From the investor's view ${ }^{2}$, the utility indifference price of the corporate bond $P$ is the price such that the investor is indifferent between holding and not holding that corporate bond, i.e.

$$
\tilde{V}(x, t)=\bar{V}(x-P, v, t) .
$$

The value function defined by (5) is already well known, so we only concentrate on the value function defined by (6). Let $\bar{\pi}=\pi \sigma_{S}$, and consider the following HJB equation Cauchy problem:

$$
\begin{cases}V_{t}(x, v, t)+\sup _{\bar{\pi} \in \Pi} \mathcal{L}^{\bar{\pi}} V(x, v, t)=0, & (x, v, t) \in(a, \infty) \times \mathbb{R}_{+} \times[0, T), \\ \left.V\right|_{t=T}=U(x+\min (v, F)), & (x, v) \in(a, \infty) \times \mathbb{R}_{+},\end{cases}
$$

where

$$
\mathcal{L}^{\bar{\pi}} V=\frac{1}{2} \bar{\pi}^{2} V_{x x}+\frac{1}{2} \sigma_{V}^{2} v^{2} V_{v v}+\rho \bar{\pi} \sigma_{V} v V_{x v}+\left(r x+\theta_{S} \bar{\pi}\right) V_{x}+\nu v V_{v} .
$$

By the dynamic programming principle and the viscosity solution argument, we have the following lemma:

Lemma 2.1 The value function $\bar{V}(x, v, t)$ defined by (6) is the viscosity solution to (8), i.e. $\bar{V}(x, v, t)=V(x, v, t)$.

Proof See the Appendix.

\footnotetext{
${ }^{2}$ There is also the utility indifference price from the firm's view, but the idea is essentially the same, so we only consider our problem from the investor's view.
} 


\subsection{Closed formulae under CARA utility function}

In order to obtain the price of corporate bonds, we need to solve the HJB equation Cauchy problem (8). It is impossible to derive the explicit solutions to (8) in most situations. However, for CARA utility functions, the explicit solution to (8) is possible, and we will derive the closed formulae for corporate bond price in this subsection.

Assumption 2.6 (the investor)

The investor has an exponential utility function:

$$
U(x)=-\exp \{-\gamma x\}, \quad \text { for } x \in \mathbb{R} \text { and } \gamma>0 .
$$

This utility function is called constant absolute risk aversion (CARA) utility function, i.e. $R_{a}(x)=-U^{\prime \prime}(x) / U^{\prime}(x)=\gamma$, which is a constant.

To solve the problem (8), first note that the optimal feedback control for the problem (8) is

$$
\begin{aligned}
\bar{\pi}^{*}(x, v, t) & =\operatorname{argmax}_{\bar{\pi} \in \mathbb{R}}\left\{\frac{1}{2} V_{x x} \bar{\pi}^{2}+\left(\rho \sigma_{V} v V_{x v}+\theta_{S} V_{x}\right) \bar{\pi}\right\} \\
& =-\frac{\rho \sigma_{V} v V_{x v}+\theta_{S} V_{x}}{V_{x x}} .
\end{aligned}
$$

Substitute (9) into (8), we obtain the Monge-Ampère equation defined on $\mathbb{R} \times \mathbb{R}_{+} \times[0, T]:$

$$
\begin{aligned}
V_{t} V_{x x} & +\frac{1}{2} \sigma_{V}^{2} v^{2}\left[V_{v v} V_{x x}-\rho^{2}\left(V_{x v}\right)^{2}\right] \\
& -\frac{1}{2} \theta_{S}^{2}\left(V_{x}\right)^{2}-\rho \theta_{S} \sigma_{V} v V_{x} V_{x v}+\nu v V_{v} V_{x x}+r x V_{x} V_{x x}=0
\end{aligned}
$$

with the terminal condition:

$$
\left.V\right|_{t=T}=-\exp \{-\gamma(x+\min (v, F))\} .
$$

Noting that the variables are separable in the terminal condition (11), we can conjugate the solution in the multiple form. Then by the Cole-Hopf transformation and the Green's function, we solve the problem (10) (11) as follows:

Theorem 2.2 If the utility function is given by Assumption 2.6, the value function defined by (6), which is the solution to the Monge-Ampère equation Cauchy problem (10) (11), is

$$
\bar{V}(x, v, t)=-e^{-\gamma e^{r(T-t)} x}\left(\int_{0}^{\infty} G(v, t ; \xi, T) e^{\gamma\left(\rho^{2}-1\right) \min (\xi, F)} d \xi\right)^{\frac{1}{1-\rho^{2}}}
$$


where $G(v, t ; \xi, T)$ is the Green's function (the transition density) in the form:

$G(v, t ; \xi, T)=\frac{e^{-\frac{1}{2}\left(1-\rho^{2}\right) \theta_{S}^{2}(T-t)}}{\xi \sigma_{V} \sqrt{2 \pi(T-t)}} \exp \left\{-\frac{\left[\ln \frac{v}{\xi}+\left(\nu-\rho \theta_{S} \sigma_{V}-\frac{\sigma_{V}^{2}}{2}\right)(T-t)\right]^{2}}{2 \sigma_{V}^{2}(T-t)}\right\}$.

Proof See the Appendix.

Now that we obtain the value function $\bar{V}(x, v, t)$, the utility indifference price of corporate bonds can be derived as follows:

Corollary 2.3 If the utility function is given by Assumption 2.6, then the utility indifference price of the corporate bond under the contract's Assumption 2.3 is

$$
P(v, t)=\frac{e^{-r(T-t)}}{\gamma\left(\rho^{2}-1\right)} \ln \left(\int_{0}^{\infty} \bar{G}(v, t ; \xi, T) e^{\gamma\left(\rho^{2}-1\right) \min (\xi, F)} d \xi\right),
$$

where

$$
\bar{G}(v, t ; \xi, T)=e^{\frac{1}{2}\left(1-\rho^{2}\right) \theta_{S}^{2}(T-t)} G(v, t ; \xi, T) .
$$

Proof For the exponential utility function, it is well known that the value function (5) is

$$
\tilde{V}(x, t)=-e^{-\gamma e^{r(T-t)} x} e^{-\frac{1}{2} \theta_{S}^{2}(T-t)} .
$$

Combining the above with the value function $\bar{V}(x, v, t)$ obtained in Theorem 2.2, the pricing formula (14) follows from Definition 2.1.

There are some new features of the utility indifference price of corporate bonds. Firstly, the price depends on the individual's utility function, which means different investors will have distinctive views on the the same corporate bond. Secondly, the pricing rule is no longer linear, which reflects the investor's attitude towards risk. However, based on the pricing formula (14), the specific nonlinear relation can be obtained (See Section 3), and this will facilitate us to analyze our structural model for credit risk. Finally the investor's initial wealth does not influence the valuation. The reason behind this is due to the choice of CARA utility functions. Nevertheless, the stocks can influence the price of corporate bonds indirectly by the correlation parameter $\rho$, and this motivates us to construct the hedging strategy for credit risk by trading the stocks (See Section 3). The more details of the utility indifference valuation distinctive from the classical structural models will be presented in Section 5, and the corresponding numerical results will be provided in Section 6 . 


\subsection{Closed formulae under other utility functions}

We will consider the pricing of corporate bonds under other utility functions in this subsection. Checking the solution procedure for the exponential utility case, we observe that the crucial assumption is in fact not exponential functions but their variable-separable property. Therefore in this subsection we will consider the utility function of the form $U(x, v)=h(x) g(v)$, where $h(x)$ is the utility defined on the wealth and $g(v)$ is the utility defined on the payoff of corporate bonds. When $h(x)$ is a power function, Zariphopoulou (2001) considered a similar problem, where she regarded $v$ as a stochastic factor.

Assumption 2.7 (the investor)

The investor has a variable-separable utility function: $U(x, v)=h(x) g(v)$, where $g \in L^{2}$, i.e. $g$ is square-integrable, and $h(x)$ is of the type:

(1) Exponential type $U(x, v)=-e^{\gamma x} g(v)$ for $x \in \mathbb{R}$ and $\gamma>0$;

(2) Power type $U(x, v)=\frac{x^{\alpha}}{\alpha} g(v)$ for $x \in \mathbb{R}_{+}$and $0<\alpha<1$.

The value function $\bar{V}(x, v, t)$ can be derived in a similar way to Theorem 2.2. Combining it with the value function $\tilde{V}(x, t)$, we obtain the pricing formulae for the utility functions of variable-separable type as follows:

Corollary 2.4 If the utility function is given by Assumption 2.7, then the utility indifference price of the corporate bond under the contract's Assumption 2.3 is

(1) For exponential type $U(x, v)=-e^{\gamma x} g(v)$,

$$
P(v, t)=\frac{e^{-r(T-t)}}{\gamma\left(\rho^{2}-1\right)} \ln \left(\int_{0}^{\infty} \bar{G}(v, t ; \xi, T) g(\xi)^{\rho^{2}-1} d \xi\right)
$$

where $\bar{G}(v, t ; \xi, T)$ is given by (15).

(2) For power type $U(x, v)=\frac{x^{\alpha}}{\alpha} g(v)$,

$$
P(x, v, t)=x\left\{1-\left(\int_{0}^{\infty} \hat{G}(v, t ; \xi, T) g(\xi)^{1+\frac{\rho^{2} \alpha}{1-\alpha}} d \xi\right)^{\alpha+\frac{\rho^{2} \alpha^{2}}{1-\alpha}}\right\}
$$

where

$\hat{G}(v, t ; \xi, T)=\frac{1}{\xi \sigma_{V} \sqrt{2 \pi(T-t)}} \exp \left\{-\frac{\left[\ln \frac{v}{\xi}+\left(\nu+\frac{\rho \theta_{S} \sigma_{V} \alpha}{1-\alpha}-\frac{\sigma_{V}^{2}}{2}\right)(T-t)\right]^{2}}{2 \sigma_{V}^{2}(T-t)}\right\}$. 
Proof Both solution procedures follow from Theorem 2.2 and Corollary 2.3, which first conjugate the solution in the multiply form in order to separate variables, then use the Cole-Hopf transformation to derive linear PDEs. The Green's function (See Jiang (2005)) is applied to obtain the explicit solutions finally.

\section{Default probability and hedging strategy}

In this section we will derive the default probability and the hedging strategy for corporate bonds based on the pricing formula (14) obtained in Section 2 .

\subsection{Minimal relative entropy martingale measure}

Under the framework that the underlying processes are locally bounded semimartingales and that the utility function is exponential, Delbaen et al. (2002) proved the following result by the duality argument:

$$
\sup _{\pi} E\left[-\exp \left\{-\gamma\left(X_{T}^{\pi}+P_{T}\right)\right\}\right]=-\exp \left\{-\inf _{\mathbb{Q}}\left\{H(\mathbb{Q} \mid \mathbb{P})+\gamma E^{\mathbb{Q}}\left[P_{T}\right]\right\}\right\},
$$

for some appropriate trading strategy $\pi$ and martingale measure $\mathbb{Q}$, where $H(\mathbb{Q} \mid \mathbb{P})$ is the relative entropy of $\mathbb{Q}$ with respect to $\mathbb{P}$ :

$$
H(\mathbb{Q} \mid \mathbb{P}):= \begin{cases}E\left[\frac{d \mathbb{Q}}{d \mathbb{P}} \ln \left(\frac{d \mathbb{Q}}{d \mathbb{P}}\right)\right] & \text { if } \mathbb{Q} \ll \mathbb{P} \\ \infty & \text { otherwise }\end{cases}
$$

This means that solving our optimal portfolio problem is equivalent to choosing the minimal relative entropy martingale measure. Next we will identify the martingale measure from the pricing formula (14) and will prove it is the minimal relative entropy martingale measure. By the Feynman-Kac representation, the equivalent martingale measure of the pricing formula (14) is given as follows:

Lemma 3.1 If the utility function is given by Assumption 2.6, and the interest rate $r=0^{3}$, the price process of the corporate bond $\left(P_{t}\right)_{0 \leq t \leq T}$ is a $\left(\mathbb{Q},\left\{\mathcal{F}_{t}^{V}\right\}_{0 \leq t \leq T}\right)$-martingale in the following sense:

$$
e^{\gamma\left(\rho^{2}-1\right) P_{t}}=E^{\mathbb{Q}}\left[e^{\gamma\left(\rho^{2}-1\right) P_{T}} \mid \mathcal{F}_{t}^{V}\right], \quad 0 \leq t \leq T,
$$

\footnotetext{
${ }^{3}$ This is equivalent to say, we consider the discounted underlying processes instead.
} 
where the Radon-Nikodym density $\mathbb{Q}$ is

$$
\frac{d \mathbb{Q}}{d \mathbb{P}}=\exp \left\{-\theta_{S} W_{T}^{S}-\frac{1}{2} \theta_{S}^{2} T\right\}
$$

Proof See the Appendix.

Lemma 3.1 specifies the equivalent martingale measure and provides us the pricing formula in the martingale form, so any contingent claim can be valued by (18), provided that we first discount the underlying process. The default probability can be regarded as the contingent claim with the final payoff $1_{\left\{V_{T} \leq F\right\}}$ and with interest rate $r=0$. Therefore by Lemma 3.1, we can derive the default probability under the utility indifference valuation framework as follows:

Corollary 3.2 If the utility function is given by Assumption 2.6, the default probability under the contract's Assumption 2.3 is

$$
p(v, t)=\frac{1}{\gamma\left(\rho^{2}-1\right)} \ln \left(e^{\gamma\left(\rho^{2}-1\right)} \int_{0}^{F} \bar{G}(v, t ; \xi, T) d \xi+\int_{F}^{\infty} \bar{G}(v, t ; \xi, T) d \xi\right)
$$

where $\bar{G}$ is the same as (15) except that $\theta_{S}=\mu / \sigma_{S}$.

Proof Since the default probability can be regarded as the contingent claim with the final payoff $1_{\left\{V_{T} \leq F\right\}}$, by Lemma 3.1, the default probability $p$ satisfies

$$
e^{\gamma\left(\rho^{2}-1\right) p(v, t)}=E^{\mathbb{Q}}\left[e^{\gamma\left(\rho^{2}-1\right) 1_{\left\{V_{T} \leq F\right\}}} \mid \mathcal{F}_{t}^{V}\right]
$$

As the argument in Lemma 3.1, if let $\bar{p}(v, t)=e^{\gamma\left(\rho^{2}-1\right) p(v, t)}$, then by the Feynman-Kac representation (See Karatzas and Shreve (1991)):

$$
\left\{\begin{array}{l}
\bar{p}_{t}+\frac{1}{2} \sigma_{V}^{2} v^{2} \bar{p}_{v v}+\left(\nu-\rho \theta_{S} \sigma_{V}\right) v \bar{p}_{v}=0 \\
\left.\bar{p}\right|_{t=T}=\exp \left\{\gamma\left(\rho^{2}-1\right) H(F-v)\right\}
\end{array}\right.
$$

where $H(F-v)$ is the Heaviside function. The problem (21) can be solved by employing the Green's function, and then the default probability $p$ can be obtained from $\bar{p}$.

Next we will identify that the equivalent martingale measure $\mathbb{Q}$ is just the minimal relative entropy measure based on the following characteristic result of Rheinländer (2005). 
Theorem 3.3 (Rheinländer (2005)) Let $\left(S_{t}\right)_{0 \leq t \leq T}$ be a locally bounded semimartingale under $\mathbb{P}$. If $\mathbb{Q}$ has finite relative entropy and the Radon-Nikodym density is of the form:

$$
\frac{d \mathbb{Q}}{d \mathbb{P}}=\exp \left\{c+\int_{0}^{T} \eta_{t} d S_{t}\right\}
$$

with $\int_{0}^{T} \eta_{t}^{2} d[S]_{t}$ in the Orlicz space $L_{\text {exp }}(\mathbb{P})$ generated by the Young function $\exp (\cdot)$, where $c$ is a constant and $\left(\eta_{t}\right)_{0 \leq t \leq T}$ is a predictable process, then $\mathbb{Q}$ is the minimal relative entropy measure.

Proposition 3.1 The equivalent martingale measure $\mathbb{Q}$ defined by (19) is the minimal relative entropy measure.

Proof Let us first recall the definitions of Young function and Orlicz space.

A function $A(\cdot)$ is called Young function if it is convex, strictly increasing on $[0, \infty)$ such that $A(0)=0$. Orlicz space $L_{A}(\mathbb{P})$ is the set of all measurable functions $u(\cdot)$ for which there exists a positive $\lambda$ such that

$$
\int A\left(\frac{|u(x)|}{\lambda}\right) d P<\infty
$$

Since $H(\mathbb{Q} \mid \mathbb{P})=\frac{1}{2} \theta_{S}^{2} T, \mathbb{Q}$ has finite relative entropy. The Radon-Nikodym density (19) can be rewritten as

$$
\begin{aligned}
\frac{d \mathbb{Q}}{d \mathbb{P}} & =\exp \left\{-\theta_{S} W_{T}^{S}-\frac{1}{2} \theta_{S}^{2} T\right\} \\
& =\exp \left\{\frac{\mu^{2}}{2 \sigma_{S}^{2}} T\right\} \exp \left\{\int_{0}^{T}\left(-\frac{\mu}{\sigma_{S}^{2}} S_{t}^{-1}\right) d S_{t}\right\}
\end{aligned}
$$

Choose $c=\frac{\mu^{2}}{2 \sigma_{S}^{2}} T$ and $\eta_{t}=-\frac{\mu}{\sigma_{S}^{2}} S_{t}^{-1}$. It is obvious that $\int_{0}^{T} \eta_{t}^{2} d[S]_{t} \in L_{\exp }(\mathbb{P})$, since $\int_{0}^{T} \eta_{t}^{2} d[S]_{t}=\theta_{S}^{2} T$, which is a constant. Theorem 3.3 now implies that $\mathbb{Q}$ is the minimal relative entropy measure.

\subsection{Exponential hedging of credit risk}

Generally speaking, credit risk can not be hedged, since there is no corresponding underlying assets for hedging. Notwithstanding, based on the pricing formula in the martingale form in Lemma 3.1 and the fact that the value of the stocks is correlated with the value of the firm's assets, we can construct the hedging strategy by trading the stocks to hedge the credit risk of corporate bonds. 
Our aim is to find a hedging strategy $\left(\pi_{t}\right)_{0 \leq t \leq T}$ such that the wealth process $\left(X_{t}^{\pi}\right)_{0 \leq t \leq T}$ approaches the price process of the corporate bond $\left(P_{t}\right)_{0 \leq t \leq T}$ in some sense. The first difficulty is that the investor can only trade the stocks $S$ and the risk-free bonds rather than the firm's assets $V$. However, since the driving noise of stocks $\left(W_{t}^{S}\right)_{0 \leq t \leq T}$ is correlated with the driving noise of the firm's assets $\left(W_{t}^{V}\right)_{0 \leq t \leq T}$, the hedging is possible by trading the stocks, and to this end we resort to the orthogonal decomposition:

$$
W_{t}^{V}=\rho W_{t}^{S}+\sqrt{1-\rho^{2}} W_{t}^{\perp} \text { for } 0 \leq t \leq T,
$$

where $\left(W_{t}^{S}\right)_{0 \leq t \leq T}$ and $\left(W_{t}^{\perp}\right)_{0 \leq t \leq T}$ are the orthogonal Brownian motions ${ }^{4}$. The second difficulty is that both wealth process $\left(X_{t}^{\pi}\right)_{0 \leq t \leq T}$ and price process $\left(P_{t}\right)_{0 \leq t \leq T}$ are not martingales under the original measure $\mathbb{P}$. To overcome this difficulty, we can use Lemma 3.1 which claims that the exponential of $P_{t}$ is a martingale in some sense. We will construct the hedging strategy as follows.

On one hand, the wealth equation (4) under the assumption in Lemma 3.1 can be simplified to

$$
\left\{\begin{array}{l}
d X_{t}^{\pi}=\pi_{t} \mu d t+\pi_{t} \sigma_{S} d W_{t}^{S}, \quad t \in(0, T] \\
X_{0}=x
\end{array}\right.
$$

If define the equivalent martingale measure $\mathbb{Q}$ by $(19), \bar{W}_{t}^{S}=W_{t}^{S}+\theta_{S} t$ is Brownian motion under $\mathbb{Q}$ by the Girsanov theorem (See Karatzas and Shreve (1991)), and therefore the wealth process $X^{\pi}$ is a $\left(\mathbb{Q},\left\{\mathcal{F}_{t}^{S}\right\}_{0 \leq t \leq T}\right)$-martingale:

$$
X_{t}^{\pi}=X_{0}+\int_{0}^{t} \pi_{s} \mu d s+\int_{0}^{t} \pi_{u} \sigma_{S} d W_{u}^{S}=X_{0}+\int_{0}^{t} \pi_{u} \sigma_{S} d \bar{W}_{u}^{S} .
$$

On the other hand, the price process $e^{\gamma\left(\rho^{2}-1\right) P_{t}}$ is a $\left(\mathbb{Q},\left\{\mathcal{F}_{t}^{V}\right\}_{t \geq 0}\right)$-martingale by Lemma 3.1. By the martingale representation (See Karatzas and Shreve (1991)), there exists a unique $\mathcal{F}_{t}^{V}$-adapted process $H$ such that $E^{\mathbb{Q}}\left[\int_{0}^{T} H_{t}^{2} d t\right]<$ $\infty$ and

$$
e^{\gamma\left(\rho^{2}-1\right) P_{t}}=e^{\gamma\left(\rho^{2}-1\right) P_{0}}+\int_{0}^{t} H_{u} d \bar{W}_{u}^{V} .
$$

Since $e^{\gamma\left(\rho^{2}-1\right) P_{t}}$ is also square-integrable, we can derive the specific form of $H_{t}$. In fact, letting $\bar{P}_{t}=e^{\gamma\left(\rho^{2}-1\right) P_{t}}$, apply Itô's lemma to $\bar{P}_{t}=\bar{P}\left(V_{t}, t\right)$ and by the equation (21):

$$
\begin{aligned}
d \bar{P}\left(V_{t}, t\right) & =\left(\frac{\partial \bar{P}}{\partial t}+\frac{1}{2} \sigma_{V}^{2} V_{t}^{2} \frac{\partial^{2} \bar{P}}{\partial v^{2}}+\left(\nu-\rho \theta_{S} \sigma_{V}\right) V_{t} \frac{\partial \bar{P}}{\partial v}\right) d t+\sigma_{V} V_{t} \frac{\partial \bar{P}}{\partial v} d \bar{W}_{t}^{V} \\
& =\sigma_{V} V_{t} \frac{\partial \bar{P}}{\partial v} d \bar{W}_{t}^{V}=\gamma\left(\rho^{2}-1\right) \sigma_{V} V_{t} e^{\gamma\left(\rho^{2}-1\right) P_{t}} \frac{\partial P}{\partial v} d \bar{W}_{t}^{V} .
\end{aligned}
$$

\footnotetext{
${ }^{4}$ This implicitly assumes that the firm's asset value contains more information than the stocks. i.e. $\mathcal{F}_{t}^{S} \subset \mathcal{F}_{t}^{V}=\mathcal{F}_{t}$. Therefore the credit risk cannot be fully hedged.
} 
So

$$
H_{t}=\gamma\left(\rho^{2}-1\right) \sigma_{V} V_{t} e^{\gamma\left(\rho^{2}-1\right) P\left(V_{t}, t\right)} \frac{\partial P}{\partial v}\left(V_{t}, t\right)
$$

The hedging strategy $\left(\pi_{t}\right)_{0 \leq t \leq T}$ can be derived by comparing (24) with (25), and in fact it is optimal in $L^{2}$ sense. To conclude this section, we give the hedging strategy for credit risk as the following theorem:

Theorem 3.4 If the utility function is given by Assumption 2.6, the hedging strategy $\left(\pi_{t}\right)_{0 \leq t \leq T}$ for the corporate bond, which is specified by the contract's Assumption 2.3, is as follows:

$$
\left\{\begin{array}{l}
X_{0}=e^{\gamma\left(\rho^{2}-1\right) P_{0}}=e^{\gamma\left(\rho^{2}-1\right) P\left(V_{0}, 0\right)}, \\
\pi_{t}=\frac{\rho H_{t}}{\sigma_{S}}=\frac{\gamma \rho\left(\rho^{2}-1\right) \sigma_{V}}{\sigma_{S}} V_{t} e^{\gamma\left(\rho^{2}-1\right) P\left(V_{t}, t\right)} \frac{\partial P}{\partial v}\left(V_{t}, t\right) \quad \text { for } \quad 0 \leq t \leq T,
\end{array}\right.
$$

and this strategy is such that the following infinitum is attained:

$$
\inf _{\left(\pi_{t}\right)_{0 \leq t \leq T}}\left\{\left\|E^{\mathbb{Q}}\left[e^{\gamma\left(\rho^{2}-1\right) P_{t}} \mid \mathcal{F}_{t}^{S}\right]-X_{t}^{\pi}\right\|_{2}: e^{\gamma\left(\rho^{2}-1\right) P} \in \mathcal{H}^{2} ; X^{\pi} \in \mathcal{H}_{S}^{2}\right\}
$$

where $\mathcal{H}_{S}^{2}$ is the Banach space of the continuous and $\left(\mathbb{Q},\left\{\mathcal{F}_{t}^{S}\right\}_{0 \leq t \leq T}\right)$-martingales with the norm:

$$
\left\|X^{\pi}\right\|_{2}=\left\{E^{\mathbb{Q}}\left[\sup _{0 \leq t \leq T}\left|X_{t}^{\pi}\right|^{2}\right]\right\}^{\frac{1}{2}}
$$

and $\mathcal{H}^{2}$ is the same as $\mathcal{H}_{S}^{2}$ except that the martingales are $\mathcal{F}_{t}$-adapted.

Proof The proof is just based on the above argument. In fact by the orthogonal decomposition (22), the martingale representation (25) can be rewritten as

$$
e^{\gamma\left(\rho^{2}-1\right) P_{t}}=e^{\gamma\left(\rho^{2}-1\right) P_{0}}+\int_{0}^{t} \rho H_{u} d \bar{W}_{u}^{S}+\int_{0}^{t} \sqrt{1-\rho^{2}} H_{u} d W_{u}^{\perp} .
$$

The projection of $e^{\gamma\left(\rho^{2}-1\right) P_{t}}$ on the closed subspace $\mathcal{H}_{S}^{2}$ is

$$
\begin{aligned}
E^{\mathbb{Q}}\left[e^{\gamma\left(\rho^{2}-1\right) P_{t}} \mid \mathcal{F}_{t}^{S}\right] & =e^{\gamma\left(\rho^{2}-1\right) P_{0}}+\int_{0}^{t} \rho H_{u} d \bar{W}_{u}^{S}+E^{\mathbb{Q}}\left[\int_{0}^{t} \sqrt{1-\rho^{2}} H_{u} d W_{u}^{\perp} \mid \mathcal{F}_{t}^{S}\right] \\
& =e^{\gamma\left(\rho^{2}-1\right) P_{0}}+\int_{0}^{t} \rho H_{u} d \bar{W}_{u}^{S}
\end{aligned}
$$

In order to minimize the distance of $X_{t}^{\pi}$ to this projection, we choose $\left(\pi_{t}\right)_{0 \leq t \leq T}$ by (27), and the minimum distance is nought. Note that $\int_{0}^{t} \sqrt{1-\rho^{2}} H_{u} d W_{u}^{\perp}$ is the credit risk which cannot be hedged by the firm's stocks. 
The hedging strategy is determined by replicating the price process of the corporate bond by the wealth process. In order to realize this, they must be considered under the same martingale measure, resulting that we make the exponential transformation of the price process of the corporate bond. This is the reason we call it the exponential hedging strategy. The hedging strategy $\left(\pi_{t}\right)_{0 \leq t \leq T}$ is inextricably linked with the correlation parameter $\rho$. In fact, when $\rho$ goes to 1 , meaning the stock value and the firm's asset value are perfectly correlated, $\left(\pi_{t}\right)_{0 \leq t \leq T}$ corresponds to the risk-neutral hedging strategy; when $\rho$ tends to 0 , meaning the stock value is independent of the firm's asset value, the credit risk of the corporate bond in this situation cannot be hedged at all.

\section{Default before maturity}

In this section, we will relax the contract's Assumption 2.3 by considering the safety covenants that give bondholders the right to reorganize the firm if the firm's asset value falls below some nominal barrier, i.e. the firstpassage-time model. Formally, the first-passage-time model associates the default event with the first hitting time of the firm's asset value process to some specific default barrier. Leung et al (2007) considered first-passage-time models in utility indifference pricing framework, while the recovery rate was not concerned in the contract when they imposed the boundary and terminal conditions of the corresponding HJB equations. In some sense, the simple payoff of the corporate bond makes the short term credit spread in their model not too much different from the classical structural models. We will consider the recovery rate in the contract, and this will lead to more flexible credit spread especially near the maturity. Compared to the foregoing situation, there are mainly two distinctive features we have to pay attention to in the first-passage-time model. First we replace the contract's Assumption 2.3 with the following safety covenant:

Assumption 4.1 (the contract of corporate bonds)

Default will occur whenever the firm's asset value process $\left(V_{t}\right)_{0 \leq t \leq T}$ falls below the default barrier, denoted by $D_{t}$. Here we model the default barrier evolving over time as follows:

$$
D_{t}=F e^{-\nu(T-t)} .
$$

The default time is defined as the following stopping time:

$$
\tau=\inf \left\{t \geq 0 \mid V_{t} \leq D_{t}\right\}
$$


and the payoff of the contract is

$$
P_{\tau \wedge T}=\omega D_{\tau} 1_{\{\tau \leq T\}}+F 1_{\{\tau>T\}},
$$

where the constant $\omega$ is the recovery rate that the firm will pay for the corporate bond's investors when default occurs.

Under the contract's Assumption 4.1, the investor's second optimal portfolio problem (6) will be replaced by (32), whereas the first one is still the same as (5).

$$
\begin{aligned}
& \bar{V}(x, v, t) \\
= & \sup _{\left(\pi_{s}\right)_{t \leq s \leq T}} E\left[U\left(1_{\{\tau>T\}}\left(X_{T}^{\pi}+P_{T}\right)+1_{\{\tau \leq T\}} e^{r(T-\tau)}\left(X_{\tau}^{\pi}+P_{\tau}\right)\right) \mid X_{t}=x, V_{t}=v\right]
\end{aligned}
$$

where $\pi, X$ and $V$ are given as before. This means if default happens, before the default time $\tau$, the investor will optimize his portfolio by holding the corporate bonds and trading the firm's stocks and the risk-free bonds, and after the default time $\tau$, the investor will put all the money he gets from the firm into the bank account and gets the risk-free interest rate $r$ only. A similar idea of how to impose the boundary condition, i.e. the case of $\tau \leq T$, can be found in Henderson (2007), when she considered the perpetual American option pricing problems.

As in Lemma 2.1, the value function $\bar{V}(x, v, t)$ in $(32)$ is the viscosity solution to the following HJB equation Dirichlet problem:

$$
\begin{cases}\bar{V}_{t}(x, v, t)+\sup _{\bar{\pi} \in \Pi} \mathcal{L}^{\bar{\pi}} \bar{V}(x, v, t)=0, & (x, v, t) \in \mathbb{R} \times\left[D_{t}, \infty\right) \times[0, T), \\ \left.\bar{V}\right|_{v=D_{t}}=-\exp \left\{-\gamma e^{r(T-t)}\left(x+\omega D_{t}\right)\right\}, & (x, t) \in \mathbb{R} \times[0, T], \\ \left.\bar{V}\right|_{t=T}=-\exp \{-\gamma(x+F)\}, & (x, v) \in \mathbb{R} \times\left[D_{t}, \infty\right),\end{cases}
$$

where the operator $\mathcal{L}^{\bar{\pi}}$ is given in (8).

Parallel to the case of default occurring at the maturity, we obtain the utility indifference price and the default probability of the corporate bond as follows:

Theorem 4.1 If the utility function is given by Assumption 2.6, under the contract's Assumption 4.1, the utility indifference price of the corporate bond is

$$
\begin{aligned}
P(v, t)= & \frac{e^{-r(T-t)}}{\gamma\left(\rho^{2}-1\right)} \ln \left(e^{\gamma\left(\rho^{2}-1\right) F} \int_{F}^{\infty} \bar{G}\left(v e^{\nu(T-t)}, t ; \xi, T\right) d \xi+\right. \\
& \left.\left.\frac{1}{2} \sigma_{V}^{2} F^{2} \int_{t}^{T} e^{\gamma\left(\rho^{2}-1\right) \omega F e^{-(\nu-r)(T-\tau)}} \frac{\partial}{\partial \xi} \bar{G}\left(v e^{\nu(T-t)}, t ; \xi, \tau\right)\right|_{\xi=F} d \tau\right)
\end{aligned}
$$


and the default probability is

$$
\begin{aligned}
p(v, t)= & \frac{1}{\gamma\left(\rho^{2}-1\right)} \ln \left(\int_{F}^{\infty} \bar{G}\left(v e^{\nu(T-t)}, t ; \xi, T\right) d \xi+\right. \\
& \left.\left.\frac{1}{2} \sigma_{V}^{2} F^{2} \int_{t}^{T} \frac{\partial}{\partial \xi} \bar{G}\left(v e^{\nu(T-t)}, t ; \xi, \tau\right)\right|_{\xi=F} d \tau\right),
\end{aligned}
$$

where $\bar{G}(v, t ; \xi, T)$ is the Green's function in the form:

$$
\begin{aligned}
\bar{G}(v, t ; \xi, T)= & \frac{1}{\xi \sigma_{V} \sqrt{2 \pi(T-t)}} \exp \left\{-\frac{\left[\ln \frac{v}{\xi}-\left(\rho \theta_{S} \sigma_{V}+\frac{\sigma_{V}^{2}}{2}\right)(T-t)\right]^{2}}{2 \sigma_{V}^{2}(T-t)}\right\} \\
& \times\left[1-\exp \left\{\frac{2 \ln \frac{F}{\xi} \ln \frac{v}{F}}{\sigma_{V}^{2}(T-t)}\right\}\right]
\end{aligned}
$$

with $\theta_{S}=(\mu-r) / \sigma_{S}$ in (34), and $\theta_{S}=\mu / \sigma_{S}$ in (35).

Proof The proof is based on Theorem 2.2 and Corollary 2.3. The only difference is that the HJB equation (33) is a half-plane problem, so the corresponding Green's function $\bar{G}(v, t ; \xi, T)$ has to be obtained by image methods (See Jiang (2005)).

In terms of the default probability, parallel to Lemma 3.1, we rely on the fact that the price process of the corporate bond $P_{t}$ is a $\left(\mathbb{Q},\left\{\mathcal{F}_{t}^{V}\right\}_{0 \leq t \leq T}\right)$ martingale when interest rate $r=0$ :

$$
e^{\gamma\left(\rho^{2}-1\right) P_{t}}=E^{\mathbb{Q}}\left[e^{\gamma\left(\rho^{2}-1\right) P_{\tau \wedge T}} \mid \mathcal{F}_{t}^{V}\right]
$$

where the equivalent martingale measure $\mathbb{Q}$ is given by $(19)$, and the default stopping time $\tau$ is given by (30). Therefore the default probability process $p_{t}$ as the contingent claim with the final payoff $1_{\{\tau \leq T\}}$ can be valued as:

$$
e^{\gamma\left(\rho^{2}-1\right) p_{t}}=E^{\mathbb{Q}}\left[e^{\gamma\left(\rho^{2}-1\right) 1_{\{\tau \leq T\}}} \mid \mathcal{F}_{t}^{V}\right] .
$$

By the Feynman-Kac representation and the Green's function, the formula (35) follows.

\section{Comparison with classical structural model}

In this section, we will compare our credit risk model with the classical structural models. In fact, the latter is just the limiting case of our model. 
The dependence structure of the pricing formulae on the parameters will also be analyzed.

To start with, we consider the case of default occurring at the maturity, i.e. the contract's Assumption 2.3. Let us recall the classical results of the structural models. For a reference, see Bielecki and Rutkowski (2002).

Lemma 5.1 Under the contract's Assumption 2.3, the price $\tilde{P}(v, t)$ and the default probability $\tilde{p}(v, t)$ of the corporate bond are

$$
\begin{aligned}
\tilde{P}(v, t) & =v N\left(-d_{1}\right)+F e^{-r(T-t)} N\left(d_{2}\right), \\
\tilde{p}(v, t) & =N\left(-d_{2}\right),
\end{aligned}
$$

where

$$
d_{1,2}=\frac{\ln \frac{v}{F}+\left(r \pm \frac{1}{2} \sigma_{V}^{2}\right)(T-t)}{\sigma_{V} \sqrt{T-t}} .
$$

We will compare the pricing formula (14) in Corollary 2.3 and the default probability formula (20) in Corollary 3.2 with the above result.

Proposition 5.1 (1) The price of the corporate bond $P(v, t)$ under the contract's Assumption 2.3 is decreasing with respect to the absolute risk aversion parameter $\gamma(\gamma>0)$, and the maximum value is

$$
\lim _{\gamma \rightarrow 0} P(v, t)=v e^{\left(\nu-\rho \theta_{S} \sigma_{V}-r\right)(T-t)} N\left(-\tilde{d}_{1}\right)+e^{-r(T-t)} F N\left(\tilde{d}_{2}\right),
$$

where

$$
\tilde{d}_{1,2}=\frac{\ln \frac{v}{F}+\left(\nu-\rho \theta_{S} \sigma_{V} \pm \frac{1}{2} \sigma_{V}^{2}\right)(T-t)}{\sigma_{V} \sqrt{T-t}} .
$$

Moreover if $\rho \theta_{S}=\theta_{V}$, where $\theta_{S}$ and $\theta_{V}$ are the Sharpe ratios of the stock value and the firm's asset value respectively, then the limit is the classical price $\tilde{P}(v, t)$, i.e.

$$
\lim _{\gamma \rightarrow 0} P(v, t)=\tilde{P}(v, t), \quad \text { if } \rho \theta_{S}=\theta_{V} .
$$

(2) In terms of the correlation parameter $\rho(0 \leq \rho \leq 1)$, the limiting cases are

$$
\begin{aligned}
& \lim _{\rho \rightarrow 0} P(v, t)=-\frac{1}{\gamma} e^{-r(T-t)} \ln \left(\int_{0}^{\infty} \bar{G}(v, t ; \xi, T) e^{-\gamma \min (\xi, F)} d \xi\right), \\
& \lim _{\rho \rightarrow 1} P(v, t)=v e^{\left(\nu-\theta_{S} \sigma_{V}-r\right)(T-t)} N\left(-\tilde{d}_{1}\right)+e^{-r(T-t)} F N\left(\tilde{d}_{2}\right) .
\end{aligned}
$$


Moreover if $\theta_{S}=\theta_{V}$, the price is $\tilde{P}(v, t)$ when $\rho$ goes to 1 , i.e.

$$
\lim _{\rho \rightarrow 1} P(v, t)=\tilde{P}(v, t), \quad \text { if } \theta_{S}=\theta_{V} .
$$

Proof See the Appendix.

From Proposition 5.1, we see that the structural model we constructed under the utility indifference valuation framework can recover the classical structural models. When the absolute risk aversion parameter $\gamma$ increases, the investor will become more risk averse and will pay less for the corporate bond. On the other hand, when $\gamma$ goes to 0 , the price will be independent of the individual preference. Moreover, if the Sharpe ratios of the stock value and firm's asset value coincide with each other, the Radon-Nikodym density (19) will reduce to the risk-neural density, resulting in the classical structural models. The correlation parameter $\rho$ is another crucial parameter, by choosing which we can also recover the classical structural models. Indeed, this has already been shown when we constructed the hedging strategy in Section 3.2 .

Proposition 5.2 (1) The default probability of the corporate bond $p(v, t)$ under the contract's Assumption 2.3 is decreasing with respect to the absolute risk aversion parameter $\gamma(\gamma>0)$, and the maximum value is

$$
\lim _{\gamma \rightarrow 0} p(v, t)=N\left(-\tilde{d}_{2}\right)
$$

Moreover

$$
\lim _{\gamma \rightarrow 0} p(v, t)=\tilde{p}(v, t), \quad \text { if } \rho \theta_{S}=\theta_{V} .
$$

(2) In terms of the correlation parameter $\rho(0 \leq \rho \leq 1)$, the limiting cases are

$$
\begin{aligned}
& \lim _{\rho \rightarrow 0} p(v, t)=-\frac{1}{\gamma} \ln \left(e^{-\gamma} \int_{0}^{F} \bar{G}(v, t ; \xi, T) d \xi\right), \\
& \lim _{\rho \rightarrow 1} p(v, t)=N\left(-\tilde{d}_{2}\right) .
\end{aligned}
$$

Moreover

$$
\lim _{\rho \rightarrow 1} p(v, t)=\tilde{p}(v, t), \quad \text { if } \theta_{S}=\theta_{V} .
$$

Proof See the Appendix.

From Proposition 5.2, we see that the dependence structure of the default probability is the same as that of the pricing formula. The only thing that 
deserves our attention is the dependence structure of the default probability with respect to the parameter $\gamma$, as it seems to go against our intuition. In fact, this is one special feature of our structural model distinctive from the classical structural models, as the latter have nothing to do with the parameter $\gamma$. The default probability will decrease as $\gamma$ increases. The reason behind this is that the default probability can also be regarded as a contingent claim, and the price of contingent claims, as shown in Proposition 5.1, is decreasing with respect to $\gamma$. This feature in fact reflects the nonlinearity of the pricing rule of our structural model. When $\gamma$ increases, the default probability will decrease, so the price the investor would like to pay is higher. We call this effect the default effect. On the other hand, the investor's utility will distort the price as well, meaning that he would like to pay less because of the increase of $\gamma$. We call this effect the utility effect. The latter effect is much more than the former effect, so the price will decrease.

The case of default occurring before the maturity is very similar to Proposition 5.1 and Proposition 5.2. The first-passage-time model chosen to be compared is as following: The default barrier is given as

$$
\tilde{D}_{t}=F e^{-r(T-t)}
$$

and the payoff of the contract is the same as (31), i.e.

$$
\tilde{P}_{\tau \wedge T}=\omega \tilde{D}_{\tau} 1_{\{\tau \leq T\}}+F 1_{\{\tau>T\}} .
$$

Then the corporate bond price can be derived by the Green's function. Since the results are too tedious to be shown, we would rather present the numerical results of them in the next section.

\section{$6 \quad$ Numerical results}

In this section we will present some numerical results based on the pricing formulae for the corporate bond that we obtained in Corollary 2.3 and Theorem 4.1.

First we consider the effects of the absolute risk aversion parameter $\gamma$ and the correlation coefficient $\rho$ on the value of the corporate bond $P(v, t ; \gamma, \rho)$ under the contract's Assumption 2.3, i.e. default may occur at the maturity, and under the contract's Assumption 4.1, i.e. default occurs at the firstpassage-time. They are displayed in Figure 1 and Figure 2 respectively.

In both cases when the absolute risk aversion parameter $\gamma$ increases, the value of the corporate bond $P(v, t ; \gamma)$ will decrease, which means the more 
risk averse the investor is, the less he will pay for the corporate bond. Turing to the correlation coefficient $\rho$, it is displayed that the value $P(v, t ; \rho)$ will become larger as the correlation coefficient $\rho$ between the stock value $S$ and the firm's asset value $V$ increases. However, this relation is not always true, and it depends on the choice of the parameters. In fact, as the arguments in Proposition 5.1 and Proposition 5.2, one can prove that if

$$
\frac{2 \theta_{S} e^{-r(T-t)}}{\gamma \sigma_{V} v P_{v}} \leq \rho \leq 1,
$$

the value of the corporate bond $P(v, t ; \rho)$ will increase when the correlation coefficient $\rho$ increases, and if

$$
0 \leq \rho \leq \min \left(\frac{2 \theta_{S} e^{-r(T-t)}}{\gamma \sigma_{V} v P_{v}}, 1\right)
$$

the value of the corporate bond $P(v, t ; \rho)$ will decrease when the correlation coefficient $\rho$ increases.

Furthermore, for the case of default happening at the maturity, we can see that when $\gamma$ goes to 0 , or $\rho$ goes to 1 , the price of the corporate bond will converge to the corporate bond price in the classical structural models (which is computed by the formula in Lemma 5.1):

(1) when $\gamma=0$ and $\rho \theta_{S}=0.5 \times \frac{0.2-0.05}{0.3}=\theta_{V}=\frac{0.1-0.05}{0.2}$,

(2) when $\rho=1, \theta_{S}=\frac{0.15-0.05}{0.4}=\theta_{V}=\frac{0.1-0.05}{0.2}$,

the corporate bond price in our model and in the classical structural models coincide with each other: It is 75.41 as shown in Figure 1. Turing to the case of default happening at the first-passage-time, Figure 2 shows that the price is not sensitive when $\gamma$ becomes large: The price will be around 38 when $\gamma$ is larger than 2 .

[Insert Figure 1 and Figure 2 here]

Secondly, we consider the credit spread of the corporate bond in both cases. Given the corporate bond price $P(v, t)$, the credit spread at time $t$ is computed using

$$
s(t, T)=-\frac{1}{T-t} \ln \frac{P(v, t)}{F e^{-r(T-t)}} .
$$

One feature of classical structural models is that the short term credit spread is close to zero, if the firm is not in financial distress near the maturity, 
i.e.

$$
\lim _{t \rightarrow T} s(t, T)= \begin{cases}\infty & \text { for } V_{T}<F \\ 0 & \text { for } V_{T} \geq F\end{cases}
$$

This contradicts empirical evidence (See Bielecki and Rutkowski (2002)). The reason is that default can be predicated in classical structural models if the firm's asset value is modeled as diffusion processes. More precisely, the default time $\tau$ is predictable, i.e. it can be announced by an increasing sequence of stopping times $\left(\tau_{n}\right)_{n \geq 1}$. In fact, this is one of the reasons people introduce jump-diffusion processes into structural models (See Zhou (1997)), or reduce to intensity models by modeling the dynamics of default as jump processes directly (See Duffie and Singleton (1999)).

In our model, the credit spread behaves differently from classical structural models. In Table 1, we compare the credit spread for both default happening at the maturity, i.e. the Merton's model, and default happening at the firstpassage-time. The column $\rho=0.2 \gamma=10$ is computed by (14) under the contract's Assumption 2.3, which corresponds to the Merton's model. The column $\rho=0.5 \gamma=2$ is computed by (34) under the contract's Assumption 4.1, which corresponds to the first-passage-time model. In both cases, the credit spread is larger than its counterparty in the classical structural model, and it does not tend to zero as time goes to maturity.

Table 1: Comparison of the credit spread

\begin{tabular}{ccccc}
\hline time $t$ & Merton & $\rho=0.2 \gamma=10$ & first-passage-time & $\rho=0.5 \gamma=2$ \\
\hline 0 & 0.0091 & 1.5239 & 0.1055 & 0.6834 \\
0.1 & 0.0086 & 1.6329 & 0.1064 & 0.755 \\
0.2 & 0.008 & 1.7648 & 0.1045 & 0.8442 \\
0.3 & 0.0072 & 1.9202 & 0.1004 & 0.9584 \\
0.4 & 0.0063 & 2.1093 & 0.0924 & 1.1096 \\
0.5 & 0.0051 & 2.3552 & 0.0786 & 1.3193 \\
0.6 & 0.0037 & 2.6732 & 0.0564 & 1.6296 \\
0.7 & 0.0022 & 3.1165 & 0.0258 & 2.1352 \\
0.8 & 0.0007 & 3.7802 & 0.0109 & 3.1038 \\
0.9 & 0 & 4.7222 & 0 & 5.6869 \\
0.95 & 0 & 5.1223 & 0 & 7.5408 \\
\hline
\end{tabular}

Parameters: $V=100, F=80, T=1, r=0.05, \sigma_{V}=0.2, \nu=0.1$, $\sigma_{S}=0.3, \mu=0.2, \omega=0.5$.

More comparison results are shown in Figure 3 for the contract's Assumption 2.3 and Figure 4 for the contract's Assumption 4.1. We can see that in both cases, the credit spread does not necessarily go to zero as time goes 
to the maturity. Specifically, when $\gamma$ turns larger or $\rho$ turns smaller, the credit spread will increase as the time $t$ goes to the maturity $T$. The reason is that we consider the firm's assets and the stocks it issues separately. In other words, we introduced another diffusion process, which comes from the stock's value, to drive credit risk. In some sense the diffusion process from the stock value plays the role of the jump processes in jump-diffusion structural models and in reduced-form models.

\section{[Insert Figure 3 and Figure 4 here]}

Finally, in Figure 5 we compare the different recovery rates under the contract's Assumption 4.1. As shown in Figure 5, the larger the recovery rate is, the more expensive the corporate bond is. We can also see that the corporate bond price in our model is more sensitive to recovery rate compared to the classical structural model. Moreover, if the recovery rate $\omega$ goes to 1 , the value of the corporate bond will converge to the risk-free bond price. In Figure 5, it will converge to 76.0981 .

[Insert Figure 5 here]

\section{Conclusion}

We modify the classical structural models for credit risk by embedding them into the framework of optimal portfolio problems in an incomplete market. The reason of doing this, as mentioned in the introduction, is that the firm's assets cannot be traded in markets and the equivalent martingale measure is not unique. In our model, the price of the corporate bonds is determined by the indifference of the investor's two utility maximization problems. Besides the uncertainty of the firm's asset value, we introduce another diffusion process, which comes from the firm's stock value, to drive credit risk. The behavior of the credit spread in our model is different from the classical structural models due to the introduction of this diffusion process. Two new parameters are introduced into the model, namely the investor's risk aversion parameter and the correlation coefficient between the firm's assets and the stocks it issues, which results in the nonlinearity of the pricing rule. Under the Markovian framework, default occurring at the maturity and at the first-passage-time are considered, and the corresponding closed formulae are derived by solving the HJB equation Cauchy problem and the HJB equation Dirichlet problem respectively. Based on the pricing formulae, the equivalent martingale measure is identified as the relative minimal entropy 
measure and the hedging strategy is constructed by trading the firm's stocks. Finally the limiting cases are considered, which recovers the results in the classical structural models.

The type of the utility functions is quite crucial to the solvability of our problem, so we only choose the CARA utility function and the variableseparable utility function. For other utility functions such as the CRRA utility function, one has to resort to numerical solutions. Another important topic in credit risk, which is not considered in this paper, is the default correlation, which is useful for credit derivative pricing such as basket CDS and CDO valuation. Both of these will be left for the future research.

\section{Acknowledgement}

The authors thank the participants in the 2nd International Financial Risk Forum in France. The first author thanks Terry Lyons, Zhongmin Qian, Monique Jeanblanc, and especially Vicky Henderson for their valuable comments and suggestions. The second author's work is supported partly by the National Basic Research Program of China (973 Program: Grant No. 2007CB814903): credit risk analysis and credit derivative pricing. 


\section{Appendix}

\section{Proof of Lemma 2.1}

To start with, let us recall the definition of viscosity solutions.

A function $V \in C\left(\mathbb{R}^{2} \times[0, T)\right)$ is called a viscosity subsolution (supersolution) to the HJB equation in (8), if for any test function $\phi \in C^{2,1}\left(\mathbb{R}^{2} \times[0, T)\right.$, whenever $V-\phi$ attains a local maximum (local minimum) at $\left(x_{0}, v_{0}, t_{0}\right) \in$ $\mathbb{R}^{2} \times[0, T)$, we have

$$
\phi_{t}\left(x_{0}, v_{0}, t_{0}\right)+\sup _{\pi \in \Pi} \mathcal{L}^{\pi} \phi\left(x_{0}, v_{0}, t_{0}\right) \geq 0(\leq 0) .
$$

Furthermore, if $V$ is both a viscosity subsolution and a viscosity supersolution, then it is called a viscosity solution to the HJB equation in (8). In addition, if $\left.V\right|_{t=T}=U(x+\min (v, F))$, then $V$ is called a viscosity solution to the HJB equation Cauchy problem (8).

We first prove that $\bar{V}$ is a viscosity supersolution. For any test function $\phi \in C^{2,1}\left(\mathbb{R}^{2} \times[0, T)\right)$, assume $\bar{V}-\phi$ attains a local minimum at $\left(x_{0}, v_{0}, t_{0}\right) \in$ $\mathbb{R}^{2} \times[0, T)$, we must prove the second part of (43). Suppose not. Then there exists $\pi^{*} \in \Pi$ and $\theta>0$ such that

$$
\phi_{t}(x, v, t)+\mathcal{L}^{\pi^{*}} \phi(x, v, t) \geq \theta>0
$$

for $(x, v, t)$ close enough to $\left(x_{0}, v_{0}, t_{0}\right)$, i.e. $\left|(x, v, t)-\left(x_{0}, v_{0}, t_{0}\right)\right|<\delta$. By the continuity of $\bar{V}$ and $\phi$, we may also suppose

$$
(\bar{V}-\phi)(x, v, t) \geq(\bar{V}-\phi)\left(x_{0}, v_{0}, t_{0}\right) .
$$

Consider constant control $\pi_{s}=\pi^{*}$ for $t_{0} \leq s<\hat{t}$, where $\hat{t}$ is small enough such that $\left|\left(X_{s}^{\pi^{*}}, V_{s}, s\right)-\left(x_{0}, v_{0}, t_{0}\right)\right|<\delta$ and $X_{s}^{\pi^{*}}$ satisfies (4) for $t_{0} \leq s<\hat{t}$. Then (44) and (45) are also satisfied with $\left(X_{s}^{\pi^{*}}, V_{s}, s\right)$ for $t_{0} \leq s<\hat{t}$. By the Bellman principle (See Yong and Zhou (1999)) and (45),

$$
0 \geq E\left[\bar{V}\left(X_{\hat{t}}^{\pi^{*}}, V_{\hat{t}}, \hat{t}\right)-\bar{V}\left(x_{0}, v_{0}, t_{0}\right)\right] \geq E\left[\phi\left(X_{\hat{t}}^{\pi^{*}}, V_{\hat{t}}, \hat{t}\right)-\phi\left(x_{0}, v_{0}, t_{0}\right)\right] .
$$

Apply Itô's lemma to $\phi\left(X_{\hat{t}}^{\pi^{*}}, V_{\hat{t}}, \hat{t}\right)$ and by the equations (2) and (4),

$$
\begin{aligned}
& E\left[\phi\left(X_{\hat{t}}^{\pi^{*}}, V_{\hat{t}}, \hat{t}\right)-\phi\left(x_{0}, v_{0}, t_{0}\right)\right] \\
= & E\left[\int_{t_{0}}^{\hat{t}} \phi_{t}\left(X_{s}^{\pi^{*}}, V_{s}, s\right)+\mathcal{L}^{\pi^{*}} \phi\left(X_{s}^{\pi^{*}}, V_{s}, s\right) d s\right] \geq \theta\left(\hat{t}-t_{0}\right),
\end{aligned}
$$

where we have used (44) in the last inequality. This is a contradiction to (46). Therefore $\bar{V}$ is a viscosity supersolution. 
On the other hand, for any test function $\phi \in C^{2,1}\left(\mathbb{R}^{2} \times[0, T)\right)$, assume $\bar{V}-\phi$ attains a local maximum at $\left(x_{0}, v_{0}, t_{0}\right) \in \mathbb{R}^{2} \times[0, T)$, We must prove the first part of (43). Suppose not. Then there exists $\theta>0$ such that

$$
\phi_{t}(x, v, t)+\mathcal{L}^{\pi} \phi(x, v, t) \leq-\theta<0
$$

for any $\pi \in \Pi$ and $(x, v, t)$ close enough to $\left(x_{0}, v_{0}, t_{0}\right)$, i.e. $\mid(x, v, t)-$ $\left(x_{0}, v_{0}, t_{0}\right) \mid<\delta$. By the continuity of $\bar{V}$ and $\phi$, we may also suppose

$$
(\bar{V}-\phi)(x, v, t) \leq(\bar{V}-\phi)\left(x_{0}, v_{0}, t_{0}\right) .
$$

Since (4) is linear, we can choose $\hat{t}$ small enough such that $\mid\left(X_{s}^{\bar{\pi}}, V_{s}, s\right)-$ $\left(x_{0}, v_{0}, t_{0}\right) \mid<\delta$ for $t_{0} \leq s<\hat{t}$ and $X_{s}^{\bar{\pi}}$ satisfies (4) for some control $\bar{\pi} \in \Pi\left[t_{0}, \hat{t}\right]$. Then (47) and (48) are also satisfied with $\left(X_{s}^{\bar{\pi}}, V_{s}, s\right)$ for $t_{0} \leq s<\hat{t}$. By the Bellman principle and (48), we can choose $\tilde{\pi} \in \Pi[\hat{t}, T]$ such that

$$
-\frac{1}{2} \theta\left(\hat{t}-t_{0}\right) \leq E\left[\bar{V}\left(X_{\hat{t}}^{\bar{\pi}}, V_{\hat{t}}, \hat{t}\right)-\bar{V}\left(x_{0}, v_{0}, t_{0}\right)\right] \leq E\left[\phi\left(X_{\hat{t}}^{\bar{\pi}}, V_{\hat{t}}, \hat{t}\right)-\phi\left(x_{0}, v_{0}, t_{0}\right)\right] .
$$

Apply Itô's lemma to $\phi\left(X_{\hat{t}}^{\bar{\pi}}, V_{\hat{t}}, \hat{t}\right)$ and by the equations (2) and (4),

$$
\begin{aligned}
& E\left[\phi\left(X_{\hat{t}}^{\bar{\pi}}, V_{\hat{t}}, \hat{t}\right)-\phi\left(x_{0}, v_{0}, t_{0}\right)\right] \\
\leq & \sup _{\pi \in \Pi} E\left[\int_{t_{0}}^{\hat{t}} \phi_{t}\left(X_{s}^{\pi}, V_{s}, s\right)+\mathcal{L}^{\pi} \phi\left(X_{s}^{\pi}, V_{s}, s\right) d s\right] \leq-\theta\left(\hat{t}-t_{0}\right),
\end{aligned}
$$

where we have used (47) in the last inequality. This is a contradiction to (49). Therefore $\bar{V}(x, v, t)$ is a viscosity subsolution. It is obvious the terminal condition is satisfied, so $\bar{V}(x, v, t)$ is a viscosity solution to the HJB equation Cauchy problem (8).

The uniqueness of the viscosity solution follows from the comparison principle (See Evans (1998)): if $\underline{V}$ and $\bar{V}$ are respectively viscosity subsolution and viscosity supersolution and $\left.\underline{V}\right|_{t=T} \leq\left.\bar{V}\right|_{t=T}$, then $\underline{V} \leq \bar{V}$ on $\mathbb{R}^{2} \times[0, T]$.

\section{Proof of Theorem 2.2}

To solve the Monge-Ampère equation (10) with the terminal condition (11), first note that all the terms are homogeneous with respect to the order of $x$ except the last term, so we make the following transformation to cancel the last term:

$$
\bar{x}=e^{r(T-t)} x .
$$

Then the Cauchy problem (10) (11) becomes

$$
\left\{\begin{aligned}
V_{t} V_{\bar{x} \bar{x}}+ & \frac{1}{2} \sigma_{V}^{2} v^{2}\left[V_{v v} V_{\bar{x} \bar{x}}-\rho^{2}\left(V_{\bar{x} v}\right)^{2}\right] \\
& -\frac{1}{2} \theta_{S}^{2}\left(V_{\bar{x}}\right)^{2}-\rho \theta_{S} \sigma_{V} v V_{\bar{x}} V_{\bar{x} v}+\nu v V_{v} V_{\bar{x} \bar{x}}=0 \\
\left.V\right|_{t=T}= & -\exp \{-\gamma(\bar{x}+\min (v, F))\}
\end{aligned}\right.
$$


Secondly, we conjugate the solution in the multiple form:

$$
V(\bar{x}, v, t)=-e^{-\gamma(\bar{x}+W(v, t))} .
$$

Then $W(v, t)$ satisfies the following semilinear PDE:

$$
\left\{\begin{array}{l}
W_{t}+\frac{1}{2} \sigma_{V}^{2} v^{2} W_{v v}+\left(\nu-\rho \theta_{S} \sigma_{V}\right) v W_{v}+\frac{1}{2} \gamma\left(\rho^{2}-1\right) \sigma_{V}^{2} v^{2}\left(W_{v}\right)^{2}+\frac{\theta_{S}^{2}}{2 \gamma}=0 \\
\left.W\right|_{t=T}=\min (v, F) .
\end{array}\right.
$$

Next, we employ the Cole-Hopf transformation (see Evans (1998)). Conjugate the solution as $C(v, t)=e^{\gamma\left(\rho^{2}-1\right) W(v, t)}$, i.e. make the transformation:

$$
W(v, t)=\frac{1}{\gamma\left(\rho^{2}-1\right)} \ln C(v, t),
$$

then we have the following linear PDE:

$$
\left\{\begin{array}{l}
\mathcal{L} C=C_{t}+\frac{1}{2} \sigma_{V}^{2} v^{2} C_{v v}+\left(\nu-\rho \theta_{S} \sigma_{V}\right) v C_{v}-\frac{1}{2}\left(1-\rho^{2}\right) \theta_{S}^{2} C=0, \\
\left.C\right|_{t=T}=\exp \left\{\gamma\left(\rho^{2}-1\right) \min (v, F)\right\} .
\end{array}\right.
$$

Therefore the remaining task is to solve the linear PDE Cauchy problem (52). Consider the Green's function of (52) which satisfies

$$
\begin{cases}\mathcal{L} C(v, t)=0, & v \in \mathbb{R}_{+}, 0 \leq t<T \\ \left.C\right|_{t=T}=\delta(v-\xi), & v \in \mathbb{R}_{+}\end{cases}
$$

where $\xi$ is the parameter, and $\delta(\cdot)$ is the Dirac function. As the solution procedures in Jiang (2005), the Green's function $G(v, t ; \xi, T)$ is given by (13). Indeed it is just the transition density.

It is known that $G^{*}(\xi, \tau ; v, t)$, where $\xi, \tau$ are the variables, and $v, t$ are the parameters, satisfies the adjoint problem of (53):

$$
\begin{cases}\mathcal{L}^{*} G^{*}(\xi, \tau ; v, t)=0, & \xi \in \mathbb{R}_{+}, \eta>t \\ \left.G^{*}\right|_{\tau=t}=\delta(\xi-v), & \xi \in \mathbb{R}_{+}\end{cases}
$$

where $\mathcal{L}^{*}$ is the adjoint operator of $\mathcal{L}$ in the form:

$$
\mathcal{L}^{*}=-\frac{\partial}{\partial T}+\frac{1}{2} \sigma_{V}^{2} \frac{\partial}{\partial \xi^{2}} \xi^{2}-\left(\nu-\rho \theta_{S} \sigma_{V}\right) \frac{\partial}{\partial \xi} \xi-\frac{1}{2}\left(1-\rho^{2}\right) \theta_{S}^{2} .
$$


Since $C(\xi, \tau)$ satisfies $(52)$ and $G^{*}(\xi, \tau ; v, t)$ satisfies (54), consider the following integral and apply integration by parts:

$$
\begin{aligned}
& \int_{0}^{\infty} d \xi \int_{t+\epsilon}^{T-\epsilon}\left[G^{*}(\xi, \tau ; v, t) \mathcal{L} C(\xi, \tau)-C(\xi, \tau) \mathcal{L}^{*} G^{*}(\xi, \tau ; v, t)\right] d \tau \\
= & \left.\int_{0}^{\infty} G^{*} C\right|_{t+\epsilon} ^{T-\epsilon} d \xi+\left.\frac{1}{2} \sigma_{V}^{2} \int_{t+\epsilon}^{T-\epsilon} \xi^{2} G^{*} \frac{\partial C}{\partial \xi}\right|_{\xi=0} ^{\xi=\infty} d \tau \\
& -\left.\frac{1}{2} \sigma_{V}^{2} \int_{t+\epsilon}^{T-\epsilon} C \frac{\partial}{\partial \xi}\left(\xi^{2} G^{*}\right)\right|_{\xi=0} ^{\xi=\infty} d \tau+\left.\left(\nu-\rho \theta_{S} \sigma_{V}\right) \int_{t+\epsilon}^{T-\epsilon} G^{*} C \xi\right|_{\xi=0} ^{\xi=\infty} d \tau
\end{aligned}
$$

We note that when $t<\tau<T$,

$$
\mathcal{L} C=0, \quad \mathcal{L}^{*} G^{*}=0,
$$

when $\xi \rightarrow 0, \infty$,

$$
\xi^{2} G^{*} \frac{\partial C}{\partial \xi} \rightarrow 0, \quad C \frac{\partial}{\partial \xi}\left(\xi^{2} G^{*}\right) \rightarrow 0, \quad G^{*} C \xi \rightarrow 0,
$$

and when $\epsilon \rightarrow 0$,

$$
G^{*}(\xi, t+\epsilon ; v, t) \rightarrow \delta(\xi-v) .
$$

By using the foregoing results and the terminal conditions of (52), we obtain

$$
C(v, t)=\int_{0}^{\infty} G^{*}(\xi, T ; v, t) e^{\gamma\left(\rho^{2}-1\right) \min (\xi, F)} d \xi .
$$

Note that $G^{*}(\xi, T ; v, t)=G(v, t ; \xi, T)$, and change back to the original function and the original variables, we obtain the desired solution (12).

Proof of Lemma 3.1

First we rewrite (14) as follows:

$$
e^{\gamma\left(\rho^{2}-1\right) P(v, t)}=\int_{0}^{\infty} \bar{G}(\xi, T ; v, t) e^{\gamma\left(\rho^{2}-1\right) \min (\xi, F)} d \xi
$$

Substituting (15) into the equation $\mathcal{L} C=0$ in (53), we obtain $\bar{G}$ satisfying

$$
\left\{\begin{array}{l}
\bar{G}_{t}+\frac{1}{2} \sigma_{V}^{2} v^{2} \bar{G}_{v v}+\left(\nu-\rho \theta_{S} \sigma_{V}\right) v \bar{G}_{v}=0 \\
\left.\bar{G}\right|_{t=T}=\delta(v-\xi)
\end{array}\right.
$$

If define $\mathbb{Q}$ by the Radon-Nikodym density (19), by the Girsanov theorem (See Karatzas and Shreve (1991)), $\bar{W}_{t}^{S}=W_{t}^{S}+\theta_{S} t$ is Brownian motion under 
$\mathbb{Q}$ and $\left(S_{t}\right)_{0 \leq t \leq T}$ is a martingale under $\mathbb{Q}$, so $\mathbb{Q}$ is an equivalent martingale measure. Moreover, by (3), $\bar{W}_{t}^{V}=W_{t}^{V}+\rho \theta_{S} t$ is also Brownian motion under $\mathbb{Q}$ and the process $\left(V_{t}\right)_{0 \leq t \leq T}$ satisfies

$$
d V_{t}=\left(\nu d t+\sigma_{V} d W_{t}^{V}\right) V_{t}=\left(\nu-\rho \theta_{S} \sigma_{V}\right) V_{t} d t+\sigma_{V} V_{t} d \bar{W}_{t}^{V} .
$$

By the Feynman-Kac representation (See Karatzas and Shreve (1991)), $\bar{G}$ is just the transition density of the process $\left(V_{t}\right)_{0 \leq t \leq T}$ under $\mathbb{Q}$, and the right hand side of (57) equals

$$
E^{\mathbb{Q}}\left[e^{\gamma\left(\rho^{2}-1\right) \min \left(V_{T}, F\right)} \mid V_{t}=v\right]
$$

This means that for the price process of the corporate bond $\left(V_{t}\right)_{0 \leq t \leq T}$, its exponential $\exp \left\{\gamma\left(\rho^{2}-1\right) V_{t}\right\}$ is a $\left(\mathbb{Q},\left\{\mathcal{F}_{t}^{V}\right\}_{0 \leq t \leq T}\right)$-martingale.

Proof of Proposition 5.1, 5.2

We first prove Proposition 5.2. Since $\bar{p}(v, t)=e^{\gamma\left(\rho^{2}-1\right) p(v, t)}$ satisfies the Cauchy problem $(21)$, it is easily seen that $p(v, t)$ satisfies the following semilinear PDE on $\mathbb{R}_{+} \times[0, T]$ :

$$
\left\{\begin{array}{l}
p_{t}+\frac{1}{2} \sigma_{V}^{2} v^{2} p_{v v}+\left(\nu-\rho \theta_{S} \sigma_{V}\right) v p_{v}+\frac{1}{2} \gamma\left(\rho^{2}-1\right) \sigma_{V}^{2} v^{2}\left(p_{v}\right)^{2}=0 \\
\left.p\right|_{t=T}=H(F-v) .
\end{array}\right.
$$

Suppose $0 \leq \gamma_{1} \leq \gamma_{2}$, and the corresponding PDE problems are denoted by $\left(\gamma_{1}\right)$ and $\left(\gamma_{2}\right)$. Since the solution to the original Cauchy problem (21) is smooth, $\left(\gamma_{1}\right)$ and $\left(\gamma_{2}\right)$ have the classical solutions $p^{\gamma_{1}}$ and $p^{\gamma_{2}}$ in $C^{\infty}\left(\mathbb{R}_{+} \times\right.$ $[0, T])$, which are also the viscosity solutions to $\left(\gamma_{1}\right)$ and $\left(\gamma_{2}\right)$ respectively. Note that

$$
p_{t}^{\gamma_{2}}+\frac{1}{2} \sigma_{V}^{2} v^{2} p_{v v}^{\gamma_{2}}+\left(\nu-\rho \theta_{S} \sigma_{V}\right) v p_{v}^{\gamma_{2}}+\frac{1}{2} \gamma_{1}\left(\rho^{2}-1\right) \sigma_{V}^{2} v^{2}\left(p_{v}^{\gamma_{2}}\right)^{2} \geq 0,
$$

so $p^{\gamma_{2}}$ is the subsolution to the problem $\left(\gamma_{1}\right)$, and it is also the viscosity subsolution. On the other hand $p^{\gamma_{1}}$ is the viscosity supersolution to $\left(\gamma_{1}\right)$, and $\left.p^{\gamma_{2}}\right|_{t=T} \leq\left. p^{\gamma_{1}}\right|_{t=T}$. By the comparison principle (See Evans (1998)), $p^{\gamma_{2}} \leq p^{\gamma_{1}}$ on $\mathbb{R}_{+} \times[0, T]$.

To prove the limit of the solution as $\gamma \rightarrow 0$, by the Arzela-Ascoli compactness criterion (See Evans (1998)), there exists a subsequence $\gamma_{n} \rightarrow 0$ such that $p^{\gamma_{n}}$ uniformly convergent to $p$ on the compact subset of $\mathbb{R}_{+} \times[0, T]$, where $p$ satisfies

$$
\left\{\begin{array}{l}
p_{t}+\frac{1}{2} \sigma_{V}^{2} v^{2} p_{v v}+\left(\nu-\rho \theta_{S} \sigma_{V}\right) v p_{v}=0 \\
\left.p\right|_{t=T}=H(F-v)
\end{array}\right.
$$


By solving (60), we get the limiting case in (40). Similarly, we can prove the limits of the solution as $\rho \rightarrow 0$ and $\rho \rightarrow 1$ to get (41) and (42) respectively.

The proof of Proposition 5.1 is exactly the same as Proposition 5.2. We only have to derive the equation that $P(v, t)$ satisfies by considering the Cauchy problem (52). Indeed $P(v, t)$ satisfies the following semilinear PDE on $\mathbb{R}_{+} \times[0, T]$ :

$$
\left\{\begin{array}{l}
P_{t}+\frac{1}{2} \sigma_{V}^{2} v^{2} P_{v v}+\left(\nu-\rho \theta_{S} \sigma_{V}\right) v P_{v}+\frac{1}{2} \gamma\left(\rho^{2}-1\right) \sigma_{V}^{2} v^{2}\left(P_{v}\right)^{2}-r P=0 \\
\left.P\right|_{t=T}=\min (v, F)
\end{array}\right.
$$




\section{References}

[1] Avellaneda, M., Minimum-entropy Calibration of Asset Pricing Models, International Journal of Theoretical and Applied Finance 1 (1998) 447472.

[2] Bielecki, T. R., M. Jeanblanc and M. Rutkowski, Hedging of Defaultable Claims, Paris-Princeton Lectures on Mathematical Finance, Springer (2004).

[3] Bielecki, T. R. and M. Rutkowski, Credit Risk: Modeling, Valuation and Hedging, Springer (2002).

[4] Black, F. and J. Cox, Some Effects of Bond Indenture Provisions, Journal of Finance 31 (1976) 351-367.

[5] Briys, E. and F. de Varenne, Valuing Risky Fixed Rate Debt: An Extension, Journal of Financial and Quantitative Analysis 32 (1997) 239-249.

[6] Davis, M. H. A., Option Pricing in Incomplete Markets, Mathematics of Derivative Securities, Cambridge University Press (1997) 216-227.

[7] Delbaen, F., P. Grandits, T. Rheinländer, D. Samperi, M. Schweizer and C. Stricker, Exponential Hedging and Entropic Penalties, Mathematical Finance 12 (2002) 99-123.

[8] Duffie, D. and K. J. Singleton, Modeling Term Structures of Defaultable Bonds The Review of Financial Studies 12 (1999) 687-720.

[9] Evans, L. C., Partial Differential Equations, American Mathematical Society (1998).

[10] Föllmer, H. and D. Sondermann, Hedging of Non-redundant Contingent Claims, In Contribution to Mathematical Economics: Essays in Honor of G. Deberu, North-Holland (1986) 203-205.

[11] Henderson, V., Valuation of Claims on Nontraded Assets Using Utility Maximization, Mathematical Finance 12 (2002) 351-373.

[12] Henderson, V., Valuing the Option to Invest in an Incomplete Market, Mathematics and Financial Economics 1 (2007) 103-128.

[13] Henderson, V. and D. Hobson, Utility Indifference Pricing: An Overview, to appear in Indifference Pricing, Princeton University Press (2004). 
[14] Hilberink, B. and L. C. G. Rogers, Optimal capital structure and endogenous default, Finance and Stochastics 6(2) (2002) 237-263.

[15] Hobson, D., Stochastic Volatility Models, Correlations, and the qOptimal Measure, Mathematical Finance 14 (2004) 537-556.

[16] Hodges, S. and A. Neuberger, Optimal Replication of Contingent Claims under Transaction costs, Review of Future Markets 8 (1989) 222-239.

[17] Jarrow, R., and S. Turnbull, Pricing Derivatives on Financial Securities Subject to Credit Risk, Journal of Finance 50 (1995) 53-86.

[18] Jiang, L. S., Mathematical Modeling and Methods of Option Pricing, World Scientific Publishing Co. (2005).

[19] Lando D., On Cox Processes and Credit-risky Securities, Review of Derivatives Research 2 (1998) 99-120.

[20] Leung, T., S. Sircar and T. Zariphopoulou, Credit Derivatives and Risk Aversion, to appear in Advances in Econometrics, Elsevier Science. (2007)

[21] Longstaff, F. and E. Schwartz, A Simple Approach to Valuing Risky Fixed and Floating Rate Debt, Journal of Finance 50 (1995) 789-819.

[22] Karatzas, I. and S. E. Shreve, Brownian Motion and Stochastic Calculus, (2nd edition), Springer (1991).

[23] Merton, R. C., On the Pricing of Corporate Debt: The Risk Structure of Interest Rates, Journal of Finance 29 (1974) 449-470.

[24] Musiela, M. and T. Zariphopoulou, An Example of Indifference Prices under Exponential Preferences, Finance and Stochastics (2004) 229-239.

[25] Rheinländer, T., An Entropy Approach to the Stein and Stein Model with Correlation, Finance and Stochastics (2005) 399-413.

[26] Schönbucher, P. J., Credit Derivatives Pricing Models: Models, Pricing and Implementation, John Wiley and Sons (2003).

[27] Sircar, R. and T. Zariphopoulou, Utility Valuation of Credit Derivatives: Single and Two-name Cases, Advances in Mathematical Finance, Birkhauser (2007) 279-301.

[28] Sircar, R. and T. Zariphopoulou, Utility Valuation of Credit Derivatives and Application to CDOs, Working Paper (2007). 
[29] Yong, J. M. and X. Y. Zhou, Stochastic Controls: Hamiltonian Systems and HJB Equations, Springer (1999).

[30] Zariphopoulou, T., A Solution Approach to Valuation with Unhedgeable Risks, Finance and Stochastics 5 (2001) 61-82.

[31] Zhou, C. S., A Jump-diffusion Approach to Modeling Credit Risk and Valuing Defaultable Securities, Finance and Economics Discussion Series 15 (1997). 


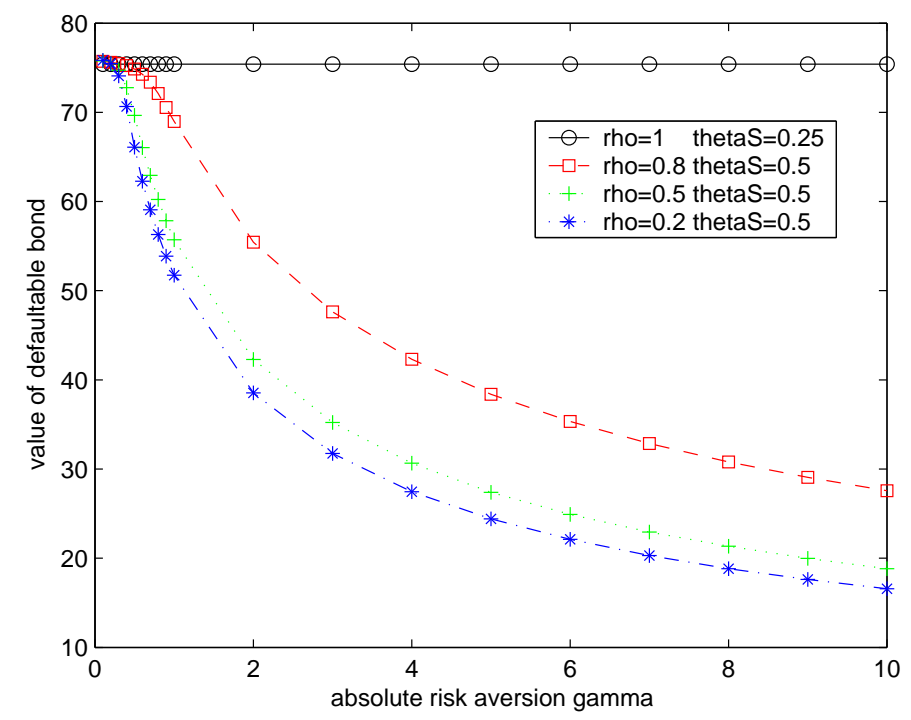

Figure 1: The value of the corporate bond in the case that default may only occur at the maturity. Parameters: $V=100, F=80, t=0, T=1, r=$ $0.05, \sigma_{V}=0.2, \nu=0.1, \sigma_{S}=0.3, \mu=0.2\left(\right.$ for $\left.\theta_{S}=0.5\right), \sigma_{S}=0.4, \mu=$ $0.15\left(\right.$ for $\left.\theta_{S}=0.25\right)$.

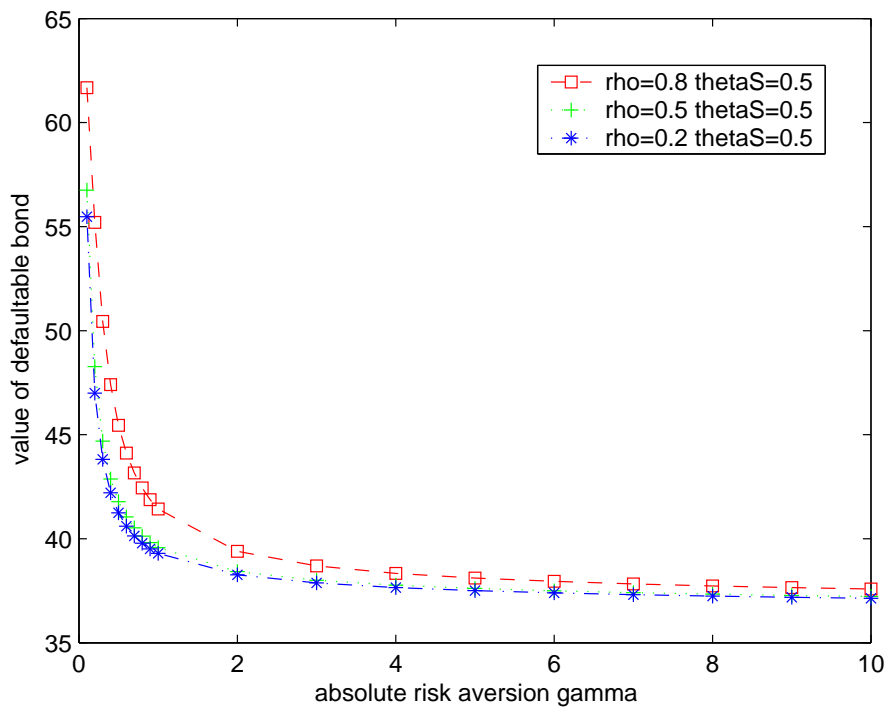

Figure 2: The value of the corporate bond in the case that default occurs at the first-passage-time. Parameters: $V=100, F=80, t=0, T=1, r=$ $0.05, \sigma_{V}=0.2, \nu=0.1, \sigma_{S}=0.3, \mu=0.2, \omega=0.5$. 


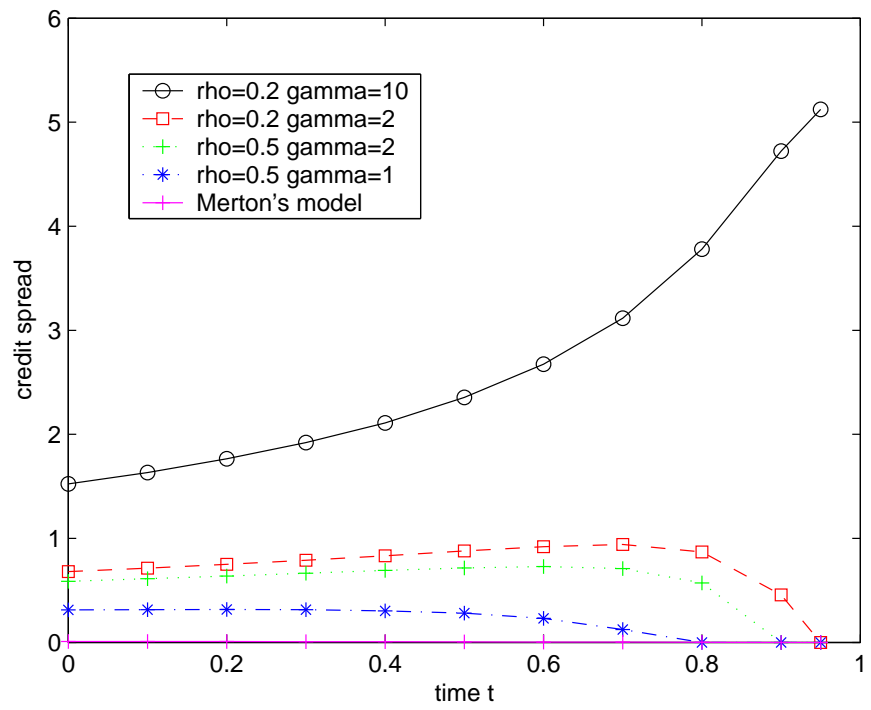

Figure 3: The credit spread in the case that default may only occur at the maturity. Parameters: $V=100, F=80, T=1, r=0.05, \sigma_{V}=0.2, \nu=$ $0.1, \sigma_{S}=0.3, \mu=0.2$.

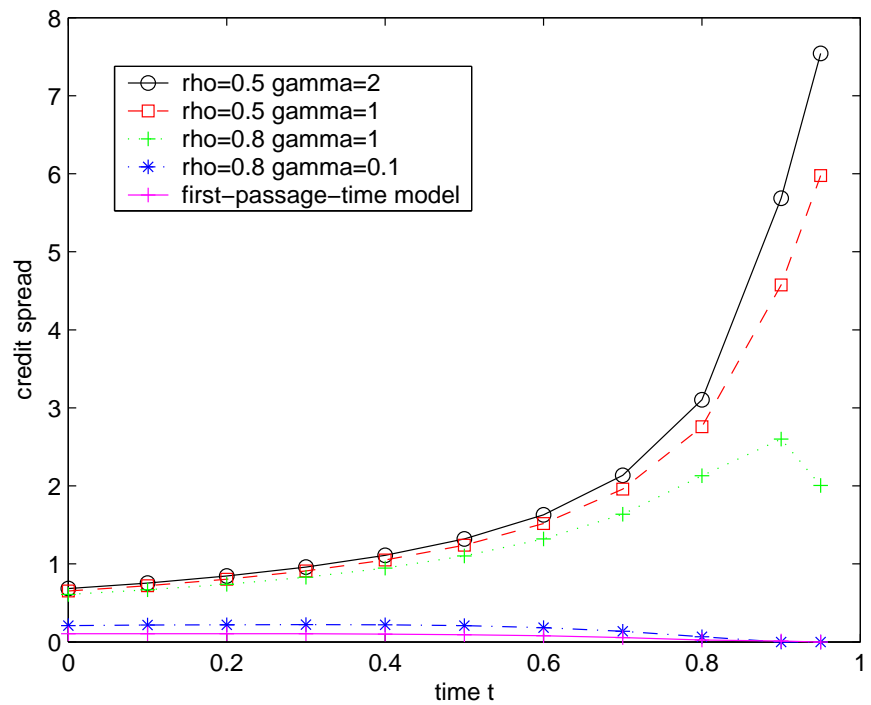

Figure 4: The credit spread in the case that default occurs at the firstpassage-time. Parameters: $V=100, F=80, T=1, r=0.05, \sigma_{V}=$ $0.2, \nu=0.1, \sigma_{S}=0.3, \mu=0.2, \omega=0.5$. 


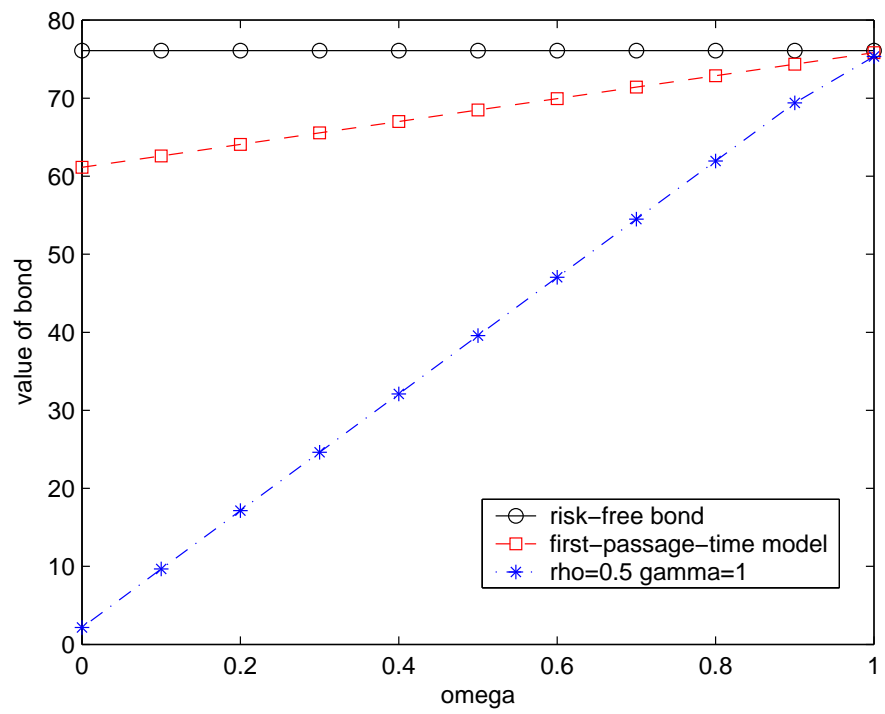

Figure 5: The value of the corporate bond in the case that default occurs at the first-passage-time. Parameters: $V=100, F=80, t=0, T=1, r=$ $0.05, \sigma_{V}=0.2, \nu=0.1, \sigma_{S}=0.3, \mu=0.2, \rho=0.5, \gamma=1$. 